\title{
THE EFFEGT OF LONGITUDINAL STRESS ON THE SHEAR STRESS AT THE BASE OF AN ICE SHEET
}

\author{
By J. F. Nye \\ (H. H. Wills Physics Laboratory, University of Bristol, Bristol, England)
}

\begin{abstract}
Robin ( 1967 ) and Budd ( 1968 , unpublished) have succeeded in connecting the variations in surface slope of an ice sheet with variations in the gradient of the longitudinal strain-rate. This paper tries to improve the theoretical basis of their work. By choice of a suitable coordinate system and suitable redefinition of the variables, Budd's formula for the basal shear stress is derived with a minimum of restrictive assumptions. The resulting formula, containing the gradient of a longitudinal stress, is thought to be of high accuracy for the two-dimensional flow of cold ice sheets, and is valid for slopes of any magnitude.

RÉsumÉ. L'effet de la contrainte longitudinale sur la contrainte de cissaillement à la base d'une nappe de glace. Robin (1967) et Budd (1968, unpublished) ont réussi à lier les variations de la pente superficielle d'une nappe de glace avec celles du gradient de la vitesse de déformation longitudinale. Cette contribution tend à améliorer la base théorique de leur travail. Par le choix d'un système adéquat de coordonnées et d'une adéquate redéfinition des variables, la formule de Budd pour la contrainte de cissaillement basale est dérivée avec un minimum d'hypothèses restrictives. La formule ainsi obtenue, contenant le gradient de la contrainte longitudinale, est supposée d'être d'une haute précision pour l'écoulement à deux dimensions de nappes de glace froide, et est valable pour des pentes de toute grandeur.

Zusammenfassung. Die Auswirkung der Längsspannung auf die Scherspannung am Grund eines Inlandeises. Robin (1967) und Budd (1968, unpublished) gelang es, einen Zusammenhang zwischen den Änderungen der Oberflächenneigung eines Inlandeises und denen des Gradienten der Längsspannung herzustellen. Dieser Aufsatz versucht, die theoretische Grundlage ihrer Arbeit zu verbessern. Durch die Wahl eines geeigneten Koordinatensystems und die Einführung von günstigen Variablen wird Budds Formel für die Scherspannung am Grund mit einem Minimum von einschränkenden Annahmen hergeleitet. Der sich ergebenden Formel, die den Gradienten der Längsspannung enthält, kann grosse Genauigkeit für das zweidimensionale Fliessen kalter Eiskalotten beigemessen werden; sie gilt für jede beliebige Neigung.
\end{abstract}

Aт the base of an ice sheet where the thickness is $h$ and the surface slope is $\alpha$ (assumed small) it has been held (Nye, 1952[a], [b]) that the shear stress $\tau_{b}$ is given approximately by

$$
\tau_{\mathrm{b}}=\rho g h \alpha,
$$

where $\rho$ is the density and $g$ is the gravitational acceleration. This formula may be justified in several different ways, which may be briefly reviewed.

(i) One way is to note that the hydrostatic pressure is much greater than the shear stresses at all levels in the ice sheet except those near the top, and to assume that it is equal to $\rho g d$, where $d$ is the depth beneath the surface. The hydrostatic stress formula $\rho g d$ is, of course, only exact when the material is a fluid, at rest, sustaining no shear stresses and having a horizontal surface. When $\alpha$ is small and non-zero, and when the material supports shear stresses, it will only hold as an approximation-and when $\alpha$ is large it will break down completely. $\alpha$ must therefore be assumed small. The rock bed is allowed to have a small, non-uniform, slope $\beta$ in the same direction as the non-uniform surface slope. On these assumptions the longitudinal stress is approximately equal to the hydrostatic stress. Equation ( $\mathrm{I}$ ) then follows (Nye, $\left.195^{2}[\mathrm{a}]\right)$ from a balance of forces, the interesting point being that $\beta$ does not appear in the final formula.

(ii) For a parallel-sided slab of ice of thickness $h$, resting on a uniform slope $\alpha$, provided all conditions are uniform down the slope, a balance of forces immediately shows that

$$
\tau_{\mathrm{b}}=\rho g h \sin \alpha .
$$

In this derivation, essentially due to Orowan (1949), there is no restriction to small $\alpha$, but if $\alpha$ is small we find Equation ( $\mathrm{I}$ ) again. Note that there is no reference to the hydrostatic stress formula. The longitudinal stress, along with everything else in the problem, including $\alpha$ and $h$, is simply assumed not to vary down the slope.

(iii) Having noted in derivation (i) that $\beta$ does not appear in Equation ( $\mathrm{I}$ ), a semiintuitive argument may be given (Nye, I952[b]) in support of the idea that Equation (I) still holds for small $\alpha$ and $\beta$ even when the bed slope $\beta$ is not in the same direction as the surface slope that is, when the strikes are not parallel). 
(iv) In an exact (numerical) calculation (Nye, 1967, p. 706) for a perfectly plastic material flowing under gravity over a horizontal base it is found that, over the greater part of the field, Equation ( $\mathrm{I}$ ) holds as a first approximation when $\alpha$ is small (and a second approximation is also given), but near the up-stream boundary the flow is disturbed from the "normal" pattern and here, even though $\alpha$ is small, Equation ( $I$ ) is totally invalid even as an approximation.

Clearly, there are conditions under which Equation (I) is a valid approximation and also conditions, even when $\alpha$ is small, where it is not. The difficulty is to formulate the precise conditions under which it is a valid first approximation, and to improve it satisfactorily. (i), (ii) and (iv) all imply different, and equally valid, sets of sufficient conditions, but the set of necessary and sufficient conditions has yet to be found.

As soon as one tries to apply Equation ( $\mathrm{I}$ ) in practice one meets the question of the interpretation of $\alpha$ and, to a lesser extent, $h$. Over what distance are these to be averaged? The derivations of the formula indicate that this distance should be at least of order $h$ and perhaps greater, but the mere fact that one cannot use very local values of $\alpha$ in the formula emphasizes its limitations.

In face of these problems there have been various attempts to improve Equation (I), and discussion has centred particularly on the role of the longitudinal stress. In all the derivations (i) to (iv) above, the longitudinal stress, in a certain sense, has been assumed uniform along the flow direction. If it has a gradient in this direction, Equation (I) will be changed. This was first emphasized by Lliboutry (1958). More recently Robin (1967), Budd (1968, unpublished) and Beitzel (in press), in interpreting field data on the surface and bed profiles of ice sheets, have concluded that longitudinal stress gradients are an important aspect of the problem. Shumskiy (196I) also has included them in his analyses.

The purpose of this paper, which arose after a very helpful discussion with Dr Budd, is to try to put the theoretical derivations which he gave on a more secure foundation. By choice of a suitable coordinate system and suitable redefinition of the variables it is possible to derive a formula for the basal shear stress that is comparatively free from restrictive assumptions, and, incidentally, is valid for any slope angle. The next step, necessary before the formula can be applied, involves a crucial assumption about the connexion between the longitudinal stress and strain-rate that may be questionable. The present treatment does not avoid this assumption, but it does remove certain doubts and difficulties from the earlier stages of the analysis. One has to bear in mind that the new term that is being calculated, and which depends on the longitudinal stress gradient, is, in some circumstances but not always, a second-order correction term to Equation (I). Therefore, particular care is necessary in making any approximations, and it becomes especially important to define all quantities precisely.

Collins's careful paper (I968), which analysed the mathematical justification for Robin's ( 1967 ) approach, has the same aims as this one, but it uses a different coordinate system with axes horizontal and vertical. Physically the two treatments are very similar (although the treatment here introduces an optional no-slip condition at the bed, which is not present in Collins's analysis) and there seems to be no disagreement between them. But the method used here is simpler. Collins does indeed refer to the possibility of taking local orthogonal axes in the surface, as is done here, but he rejects it because "the axes are curvilinear and may be initially unknown". Strictly speaking, the axes used here are rectilinear, not curvilinear. However, the equations derived are only used on the $z$-axis, which is normal to the surface, and for each surface station one sets up a new rectilinear coordinate system. Thus it is true that the coordinate directions are different for each surface station, but nevertheless the quantities in the equations are all defined with respect to rectilinear axes. There are no complications due to curvature terms in the equations of equilibrium. The fact that these axes lead to simpler equations indicates that they suit the phenomenon under investigation and thus make it easier to understand. This is a sufficient reason for using them. In some 
field applications there is an additional practical advantage in having axes referred to the upper, accessible, surface of the ice sheet. In others it may be better to use horizontal and vertical coordinates at the expense of equations that are less easy to interpret.

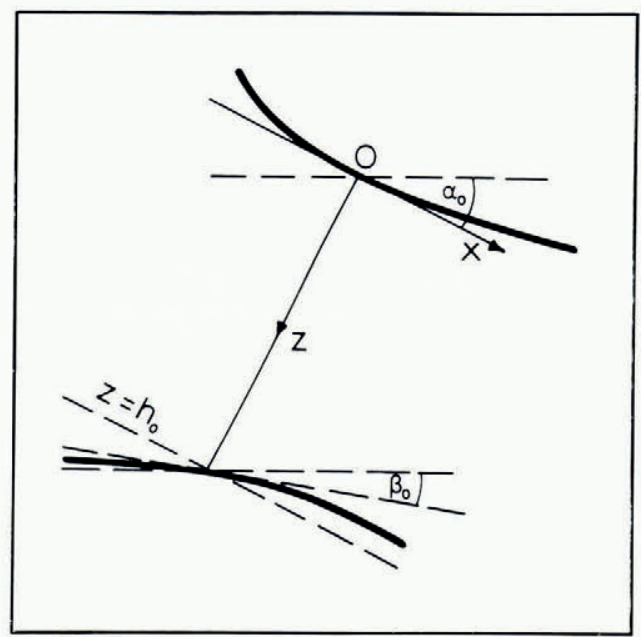

Fig. 1 . Coordinate system. Ox is tangential to the top surface at $O$.

We confine ourselves to two dimensions (plane strain). Choose an origin at some arbitrary point in the top surface (Fig. I), and choose rectilinear axes in a vertical plane, with $\mathrm{O} x$ tangential to the top surface in the direction of movement, and $\mathrm{O} z$ perpendicular to it pointing downwards. Let the angle between $\mathrm{O} x$ and the horizontal be $\alpha_{0}$, and let the slope of the bed at the point where $\mathrm{O} z$ meets it be $\beta_{0}$. Thus, although the top surface and the bed have slopes that are non-uniform, $\alpha_{0}$ and $\beta_{0}$ as defined here are constants. They need not be small and may be positive or negative. Figure I shows their positive senses. Since accelerations are negligible we may use the equations of equilibrium for the stress components $\sigma_{x}, \sigma_{z}, \tau_{x z}$ :

$$
\begin{aligned}
& \frac{\partial \sigma_{x}}{\partial x}+\frac{\partial \tau_{x z}}{\partial z}+\rho g \sin \alpha_{0}=0, \\
& \frac{\partial \tau_{x z}}{\partial x}+\frac{\partial \sigma_{z}}{\partial z}+\rho g \cos \alpha_{0}=0,
\end{aligned}
$$

for all $x$ and $z$ within the ice, and let us allow $\rho$, the density, to be a function of $x$ and $z$.

Integrate Equation (3) with respect to $z$ from $z=0$ to $z=h_{0}$, where $h_{0}$ is the thickness of the ice at $x=0$ :

for all $x$. Write

$$
\int_{0}^{h_{0}} \frac{\partial \sigma_{x}}{\partial x} \mathrm{~d} z+\int_{0}^{h_{0}} \frac{\partial \tau_{x z}}{\partial z} \mathrm{~d} z+g \sin \alpha_{0} \int_{0}^{h_{0}} \rho \mathrm{d} z=0
$$

$$
\bar{\sigma}_{x}(x)=\frac{\mathrm{I}}{h_{\mathrm{o}}} \int_{0}^{h_{0}} \sigma_{x}(x, z) \mathrm{d} z \quad \text { and } \quad \bar{\rho}(x)=\frac{\mathrm{I}}{h_{0}} \int_{0}^{h_{0}} \rho(x, z) \mathrm{d} z
$$

for all $x$. $h_{0}$, being the thickness at $x=0$, is a constant independent of $x$ (but the thickness of the ice is, of course, non-uniform and does depend on $x$ ). Therefore $\bar{\sigma}_{x}(x)$ and $\bar{\rho}(x)$, so defined, are not the mean values of $\sigma_{x}$ and $\rho$ through the thickness of the ice sheet, except at $x=0$. They are the mean values between the planes $z=0$ and $z=h_{0}$. (Where the two planes 
enter rock or air the values are undefined, but we shall see that this causes no essential difficulty.) In the first term of Equation (5) we may interchange the order of integration and differentiation (since $h_{\mathrm{o}}$ is independent of $x$ ) and hence obtain

$$
h_{0} \frac{\mathrm{d} \bar{\sigma}_{x}}{\mathrm{~d} x}+\left(\tau_{x z}\right)_{z=h_{0}}-\left(\tau_{x z}\right)_{z=\mathrm{o}}+\bar{\rho}(x) g h_{0} \sin \alpha_{0}=0
$$

for all $x$. The top surface being free, we have $\left(\tau_{x z}\right)_{z=0}=0$ at $x=0$.

It is generally supposed that when the basal ice is below the melting point it does not slip on the bed. If the basal ice does not slip, a line in the basal ice drawn parallel to the bed does not extend. Assume that the volume change of the basal ice is zero. It follows that the bed is in a direction of maximum shear strain-rate; the basal ice is deforming by simple shear. We conclude that the bed is also a direction of maximum shear stress. This would be true by symmetry if the ice were isotropic; but even if the ice were anisotropic in its flow properties it is still true, provided the flow properties have a plane of symmetry parallel or perpendicular to the bed, which seems a very plausible assumption. Now at $x=0$ the bed makes an cngle $\alpha_{0}-\beta_{0}$ with the $x$ axis, and the positive sense of $\tau_{x z}$ is opposite to that of $\tau_{b}$, the shear traction on the bed at $x=0$ (since $x$ was taken positive in the direction of motion). Therefore, for $x=\mathrm{o}$,

$$
\left(-\tau_{x z}\right)_{z=h_{\mathrm{o}}}=\tau_{\mathrm{b}} \cos 2\left(\alpha_{0}-\beta_{\mathrm{o}}\right)
$$

and, substituting in Equation (7) written for $x=0$,

$$
h_{0} \frac{\mathrm{d} \bar{\sigma}_{x}}{\mathrm{~d} x}=\tau_{\mathrm{b}} \cos 2\left(\alpha_{0}-\beta_{0}\right)-\bar{\rho} g h_{0} \sin \alpha_{0} \quad(x=0) .
$$

The term on the left in Equation (8) involves a longitudinal stress gradient. But, as we have pointed out, $\bar{\sigma}_{x}$ is not the mean $\bar{\sigma}_{x}$ through the thickness of the ice sheet, except at $x=\mathrm{o}$, and therefore $\mathrm{d} \bar{\sigma}_{x} / \mathrm{d} x$ in Equation (8) is not the gradient of the mean $\sigma_{x}$ through the thickness even at $x=0$. Likewise, $h_{0} \mathrm{~d} \bar{\sigma}_{x} / \mathrm{d} x=\mathrm{d}\left(h_{0} \bar{\sigma}_{x}\right) / \mathrm{d} x$ is not the gradient of the longitudinal force in the ice sheet at $x=0$. These distinctions arise because $\bar{\sigma}_{x}$ is defined by integration between the parallel planes $z=0$ and $z=h_{0}$ rather than between the wavy top and bottom surfaces of the ice sheet.

That the distinctions are far from trivial may be seen by considering the "hydrostatic" model we began with under heading (i), taking the base as a flat horizontal plane, and letting the density be uniform. Then $\bar{\sigma}_{x}$ equals $\frac{1}{2} \rho g h_{0}$ on $x=0$ and equals $\frac{1}{2} \rho g h_{0}+O\left(x^{2}\right)$ near $x=0$. Hence $h_{0} \mathrm{~d} \bar{\sigma}_{x} / \mathrm{d} x=\mathrm{d}\left(h_{0} \bar{\sigma}_{x}\right) / \mathrm{d} x=0$ on $x=0$. On the other hand, the longitudinal force in the ice sheet is $\frac{1}{2} \rho g h^{2}$, where $h=h(x)$ is the thickness, and the derivative of this at $x=0$ is $\rho g h_{0}(\mathrm{~d} h / \mathrm{d} x)_{0}=-\rho g h_{0} \alpha_{0}$, for small $\alpha_{0}$. Thus, in this example the left-hand side of Equation (8) is in fact zero, whereas if the left-hand side were wrongly interpreted as the longitudinal force gradient it would give a term equal to the last term on the right-hand side.

If $\alpha_{0}=\beta_{0}$ and $\mathrm{d} \bar{\sigma}_{x} / \mathrm{d} x=0$ in Equation (8) we regain Equation (2). If $\alpha_{0}$ and $\beta_{0}$ are small and $\mathrm{d} \bar{\sigma}_{x} / \mathrm{d} x=0$, as it would be if the hydrostatic stress formula were used for $\bar{\sigma}_{x}$, we regain Equation ( $\mathrm{I}$ ). Equation (8) is therefore a generalization of these two formulae.

Continuing the general analysis, let us assume that at $x=0, \partial \rho / \partial x=0$. Then, following Budd, we may differentiate Equation (4) with respect to $x$, remembering that $\alpha_{0}$ is a constant, to obtain

$$
\frac{\partial^{2} \tau_{x z}}{\partial x^{2}}+\frac{\partial^{2} \sigma_{z}}{\partial x \partial z}=0 \quad(x=0),
$$

and then integrate twice with respect to $z$, from o to $z$ and then from o to $h_{0}$ :

$$
\int_{0}^{h_{0}} \int_{0}^{z} \frac{\partial^{2} \tau_{x z}}{\partial x^{2}} \mathrm{~d} z \mathrm{~d} z+\int_{0}^{h_{0}}\left\{\frac{\partial \sigma_{z}}{\partial x}-\left(\frac{\partial \sigma_{z}}{\partial x}\right)_{z=0}\right\} \mathrm{d} z=0 \quad(x=0) .
$$


Since $\mathrm{O} x$ is tangential to the free top surface at $x=0$ it follows that $\left(\partial \sigma_{z} / \partial x\right)_{x=0, z=0}=0$, so we may write

$$
\int_{0}^{h_{0}} \int_{0}^{z} \frac{\partial^{2} \tau_{x z}}{\partial x^{2}} \mathrm{~d} z \mathrm{~d} z+\frac{\partial}{\partial x} \int_{0}^{h_{0}} \sigma_{z} \mathrm{~d} z=0 \quad(x=0) .
$$

Subtracting Equation (9) from Equation (8) we obtain

$$
\frac{\partial}{\partial x} \int_{0}^{h_{0}}\left(\sigma_{x}-\sigma_{z}\right) \mathrm{d} z=\tau_{b} \cos 2\left(\alpha_{0}-\beta_{0}\right)-\bar{\rho} g h_{0} \sin \alpha_{0}+\int_{0}^{h_{0}} \int_{0}^{z} \frac{\partial^{2} \tau x z}{\partial x^{2}} \mathrm{~d} z \mathrm{~d} z \quad(x=0) .
$$

(Since the plane $z=0$ may enter air on both sides of $x=0$, and since the plane $z=h_{0}$ will certainly enter rock on one side or other of $x=0$, it is necessary to define the $x$ derivatives in the equations at $x=0$. This may be consistently done by regarding the derivatives as limiting values obtained by letting $z \searrow 0$ and $z \nearrow h_{0}$, that is, by approaching the limits from within the ice.)

The assumptions and restrictions on which Equations (8) and (ro) are based are: (a) twodimensional flow, (b) equilibrium, (c) $\partial \rho / \partial x=0$ on $x=0$, (d) top surface free, (e) no slip on the bed, (f) no volume change in the basal ice, (g) the assumption about the anisotropy of the flow properties of the basal ice. There are no others. If the basal ice were not below the melting point, so that there was slip on the bed, the direction of maximum shear stress could cease to be parallel to the bed, and then the term $\tau_{\mathrm{b}} \cos 2\left(\alpha_{0}-\beta_{0}\right)$ would not be exactbut the term would still continue to be approximately $\tau_{\mathrm{b}}$ for small $\alpha_{0}$ and $\beta_{0}$ except at places of high longitudinal strain-rate. For cold ice in two-dimensional flow, however, all the assumptions are very plausible and, accordingly, Equations (8) and (10) are expected to be true to high accuracy in this case.

Robin bases his work on an equation similar to Equation (8) (after subtracting a hydrostatic pressure from $\bar{\sigma}_{x}$ ), while Budd uses one similar to Equation (Io), but the assumptions they make in reaching these equations and the precise meanings of some of the quantities involved are different. Collins's exact equation contains several more terms, which disappear in our coordinate system. The main points needing emphasis in Equations (8) and (IO) are: (i) the rectilinear coordinate system is defined by the upper surface and, although the surface and bed have arbitrary shapes, $\alpha_{0}$ and $\beta_{0}$ are constants; (ii) the integrations are from 0 to $h_{0}$ and not through a variable thickness $h$; (iii) $h_{0} \mathrm{~d} \bar{\sigma}_{x} / \mathrm{d} x$ is not the longitudinal force gradient; (iv) $\alpha_{0}$ and $\beta_{0}$ may be large.

The objective, which was to derive Equations (8) and (I0) as rigorously as possible, has now been reached. It remains to summarize the continuation of the argument, which is due to Budd and Robin. Write

$$
\frac{\mathrm{I}}{h_{\mathrm{o}}} \int_{\mathrm{o}}^{h_{\mathrm{o}}}\left(\sigma_{x}-\sigma_{z}\right) \mathrm{d} z=2 \bar{\sigma}_{x}^{\prime} .
$$

Budd supposes that the longitudinal strain-rate $\dot{\epsilon}$ measured at the surface is related to $\bar{\sigma}_{x}^{\prime}$ by the flow law

or better, since $\bar{\sigma}_{x}^{\prime}$ may be negative, by

$$
\dot{\epsilon}=\left(\frac{\bar{\sigma}_{x}^{\prime}}{B}\right)^{n}
$$

$$
\dot{\epsilon}=B^{-n}\left|\bar{\sigma}_{x}^{\prime}\right|^{n-1} \bar{\sigma}_{x}^{\prime}, \quad \bar{\sigma}_{x}^{\prime}=B|\dot{\epsilon}|^{(1 / n-1)} \dot{\epsilon} .
$$

This is the key assumption, and Robin makes a similar one. It may be questioned, as they recognize, because, among other difficulties, $\tau_{x z}$ as well as $\sigma_{x}^{\prime}$ is involved in a more accurate statement of the flow law. Collins discusses the circumstances in which it is a valid approximation. Here we need only note that, although a worthwhile simplification, it is on an entirely 
different footing, with regard to accuracy, from the previous assumptions listed above as (a) to (g). If it is accepted Equation (10) becomes

$$
2 h_{0} \frac{\partial}{\partial x}\left(B|\dot{\epsilon}|^{(\mathrm{r} / n-\mathrm{I})} \dot{\epsilon}\right)=\tau_{\mathrm{b}} \cos 2\left(\alpha_{0}-\beta_{\mathrm{o}}\right)-\bar{\rho} g h_{0} \sin \alpha_{0}+\int_{0}^{h_{0}} \int_{0}^{z} \frac{\partial^{2} \tau_{x z}}{\partial x^{2}} \mathrm{~d} z \mathrm{~d} z \quad(x=0) .
$$

All the quantities in Equation (I I), including $\alpha_{0}$ and $\beta_{0}$, are to be measured at $x=0$. But, since the origin was chosen arbitrarily, the equation may equally well be applied anywhere, provided of course that $\alpha_{0}$ and $\beta_{0}$ and all the other quantities are measured in the new local coordinate system. (The assumption $(\partial \rho / \partial x)_{x=0}=0$ then means simply that the ice is stratified parallel to the wavy top surface.)

To facilitate further discussion write the terms in Equation (I I) as

$$
G=\tau-\tau_{*}+\mathcal{T}
$$

( $G$ for the gradient term, $\tau$ for the basal shear stress term, $\tau_{*}$ for the shear stress as given by Equation (2), and $T$ for the last term). In his application to the Wilkes ice cap Budd averages the equivalent of Equation (I I) over a distance of $I_{5}$ to $20 \mathrm{~km}\left(\approx 20 h_{0}\right)$ at a series of points and finds that, on this scale, terms $G$ and $T$ become relatively unimportant. Thus approximately

$$
\bar{\tau}=\bar{\tau}_{*},
$$

where bars denote averages over $\approx 20 h_{0}$. For small slopes, as in this application, this is simply Equation $(\mathrm{I})$. Budd then averages over about $4 \mathrm{~km}\left(\right.$ say $\left.\approx 4 h_{0}\right)$ thus

$$
\hat{G}=\hat{\tau}-\hat{\tau}_{*}+\hat{T} \text {, }
$$

where $\hat{G}$ indicates this shorter averaging distance. He gives theoretical reasons for supposing that $\hat{T}$ is negligible. (An alternative form for $T$ in terms of $\sigma_{z}$ is given, of course, by Equation (9). According to Budd the $T$ term becomes important for fluctuations with a wavelength $3.6 h_{0}$ or less. Fluctuations on this scale are largely smoothed out by an averaging distance of $4 h_{0}$; but Budd holds that in short-distance studies, such as those by Robin and Beitzel and his own later spectrum analyses, it is essential to include the $T$ term.) Observationally he finds that, for the Wilkes ice cap,

$$
\hat{G}=\bar{\tau}_{*}-\hat{\tau}_{*},
$$

$\bar{\tau}_{*}-\hat{\tau}_{*}$ being essentially obtained from the fluctuations of $\alpha$ about the $20 h_{0}$ mean value. Hence, by combining Equations (12), (13) and (14) we have

$$
\hat{\tau}=\bar{\tau}=\bar{\tau}_{*} \text {. }
$$

The conclusion is that the basal shear stress does not fluctuate in sympathy with the surface slope $\alpha$ over distances $\approx 4 h_{0}\left(\right.$ since $\hat{\tau} \neq \hat{\tau}_{*}$ ), but it does follow the surface slope changes over distances of $\approx 20 h_{0}$ ( since $\bar{\tau}=\bar{\tau}_{*}$ ). Thus the basal shear stress fluctuates much less than $\alpha$. The observed fluctuations in $\alpha$ on the scale $\approx 4 h_{0}$ (given by $\bar{\tau}_{*}-\hat{\tau}_{*}$ ) are connected with the fluctuations $\hat{G}$ in the strain-rate gradient term.

It is to be noted that the argument in this form essentially depends on the observational fact of Equation (14). Why the ice sheet should behave in such a way that Equation (14) is true is a deeper question that does not seem to have been answered.

I am grateful to Dr I. F. Collins for his helpful comments on the first draft of this paper.

MS. received I $4_{4}$ December 1968

\section{REFERENCES}

Beitzel, J. Unpublished. The relationship of ice thicknesses and surface slopes in Queen Maud Land. [Paper read at International Symposium on Antarctic Glaciological Exploration, Hanover, N.H., U.S.A., 3-7 September 1968.] 
Budd, W. F. 1968. The longitudinal velocity profile of large ice masses. Union de Géodésie et Géophysique Internationale. Association Internationale d'Hydrologie Scientifique. Assemblée générale de Berne, 25 sept.-7 oct. 1967. [Commission de Neiges et Glaces.] Rapports et discussions, p. 58-77.

Budd, W. F. Unpublished. The dynamics of ice masses. [Ph.D. thesis, University of Melbourne, i 958, ch. 5-6.]

Collins, I. F. 1968. On the use of the equilibrium equations and flow law in relating the surface and bed topography of glaciers and ice sheets. Fournal of Glaciology, Vol. 7, No. 50, p. 199-204.

Lliboutry, L. I958. La dynamique de la Mer de Glace et la vague de I89I-95 d'après les mesures de Joseph Vallot. Union Géodésique et Géophysique Internationale. Association Internationale d'Hydrologie Scientifique. Symposium de Chamonix, $16-24$ sept. 1958, p. 125-38.

Nye, J. F. 1952[a]. A comparison between the theoretical and the measured long profile of the Unteraar glacier. Journal of Glaciology, Vol. 2, No. 12, p. $103-07$. Nye, J. F. 1952[b]. A method of calculating the thicknesses of the ice-sheets. Nature, Vol. 169, No. 4300 ,
p. 529-30.

Nye, J. F. 1967. Plasticity solution for a glacier snout. Fournal of Glaciology, Vol. 6, No. 47, p. 695-715.

Orowan, E. 1949. [The flow of ice and other solids.] (In Joint meeting of the British Glaciological Society, the British Rheologists' Club and the Institute of Metals. Fournal of Glaciology, Vol. 1, No. 5, p. 231-40.)

Robin, G. de Q. 1967 . Surface topography of ice sheets. Nature, Vol. 21 5 , No. 5105, p. 1029-32.

Shumskiy, P. A. I961. On the theory of glacier motion. Union Géodésique et Géophysique Internationale. Association Internationale d'Hydrologie Scientifique. Assemblée générale de Helsinki, 25-7-6-8 196o. Colloque sur la glaciologie antarctique, p. $142-49$. 